OPEN ACCESS

Edited by: Jacqueline Krim North Carolina State University, United States

Reviewed by: Hyun-Joon Kim, Kyungpook National University, South Korea Martin H. Müser,

Saarland University, Germany

*Correspondence:

Noshir S. Pesika npesika@tulane.edu

Specialty section:

This article was submitted to

Tribology,

a section of the journa

Frontiers in Mechanical Engineering

Received: 13 March 2019 Accepted: 06 June 2019

Published: 21 June 2019

Citation:

Pashazanusi L, Kristiansen K, Li S,

Tian Y and Pesika NS (2019) Role of Interfacial Water and Applied Potential on Friction at $\mathrm{Au}(111)$ Surfaces.

Front. Mech. Eng. 5:39. doi: 10.3389/fmech.2019.00039

\section{Role of Interfacial Water and Applied Potential on Friction at Au(111) Surfaces}

\author{
Leila Pashazanusi ${ }^{1}$, Kai Kristiansen ${ }^{2}$, Shaowei Li $^{3}$, Yu Tian ${ }^{3}$ and Noshir S. Pesika ${ }^{\text {* }}$ \\ ${ }^{1}$ Department of Chemical and Biomolecular Engineering, Tulane University, New Orleans, LA, United States, ${ }^{2}$ Department of \\ Chemical Engineering, University of California, Santa Barbara, Santa Barbara, CA, United States, ${ }^{3}$ State Key Laboratory of \\ Tribology, Tsinghua University, Beijing, China
}

The tribological properties between an AFM tip and a Au(111) surface in an aqueous environment is influenced by an applied electrical potential. Using lateral force microscopy, we measure the resulting friction force, while simultaneously applying a pre-determined electrical potential on the Au surface via a three-electrode setup. Applying a positive potential to the Au surface forms an interfacial water layer at the $\mathrm{Au} / \mathrm{electrolyte}$ interface, which sharply increases friction. However, when an anodic potential is applied, lower friction forces are measured. The potential dependent friction is observed on ultra-smooth gold surfaces as well as Au surfaces with larger roughness. An increase in the ionic strength of the electrolyte is found to lower friction. The use of an aqueous $\mathrm{NaOH}$ solution is found to lower the critical potential at which the friction sharply increases. Normal force curves are also measured as a function of approach velocity. The normal force linearly increases as the approach velocity increases in agreement with a drainage model. These results provide valuable insight into the effect of applied electrical potentials on the properties of water at charged surfaces and can potentially impact a wide range of fields including tribology, micro-electro-mechanical systems (MEMS), energy storage devices, fuel cells, and catalysis.

Keywords: nanotribology, interfacial (bound) water, electric field, hydrogen bonding, solid electrolyte interface (SEI)

\section{INTRODUCTION}

The study of molecular interactions between surfaces and colloids has been of great interest (Hugel and Seitz, 2001; Israelachvili, 2011; Balzer et al., 2013; Pashazanusi et al., 2017a) for several decades. A significant number of studies have been conducted aiming to better understand the tribological behavior of interfaces at the molecular scale (Mo et al., 2009; Schirmeisen and Schwarz, 2009; Balzer et al., 2013; Pérez, 2014; Weymouth et al., 2014). However, there are still unexplained phenomena and a clear understanding of nanoscale friction is yet to be achieved (Kim and Kim, 2009). AFM is a promising tool for acquiring diverse tribological information and understanding underlying mechanisms (Meyer et al., 1989; Overney and Meyer, 1993; Carpick and Salmeron, 1997). The ability to control and manipulate the frictional properties of a system at the nanoscale has resulted in numerous tribological studies (Park, 2011; Greiner et al., 2012; Gallagher et al., 2016). Controlling small-scale friction during sliding has potential application in a variety of areas 
including nano/micro-electro-mechanical systems (NEMS/MEMS) (Kim et al., 2007; Achanta and Celis, 2015). The application of external stimuli, which enables reversible modification of interfacial properties, is an effective strategy to control and tune friction (Binggeli et al., 1993; Park et al., 2006; Karuppiah et al., 2009). Particularly, application of the electrical potential has been shown to influence the tribological behavior at interfaces in various solutions (Labuda et al., 2011; Sweeney et al., 2012; Strelcov et al., 2015).

Within a few nanometers away from an electrode surface, water rearranges into a highly confined structure (Kim et al., 2003; Khan et al., 2010; Velasco-Velez et al., 2014), showing unique physical properties different from those of bulk water (Lee and Rossky, 1994; Toney et al., 1994; Dhinojwala and Granick, 1997; Raviv and Klein, 2002). For instance, the effective viscosity of water layers extended up to $5 \mathrm{~nm}$ away from an electrode surface has been found to increase dramatically upon applying a positive potential (Antognozzi et al., 2001; Xie et al., 2009; Guriyanova et al., 2011; Plausinaitis et al., 2014). Guriyanova et al. (2011) reported that the effective viscosity of water layers at positively charged electrode surfaces can be on the order of $10^{6}-10^{7}$ larger compared to bulk water. The influence of the highly ordered and confined water molecules on frictional properties of an electrode in the presence of an applied electrical potential have been the subject of several studies (Valtiner et al., 2012; Dhopatkar et al., 2016; Pashazanusi et al., 2017b). Dhopatkar et al. (2016) investigated the effect of a highly confined "ice-like" water layer on the friction of two surfactant coated surfaces sliding against each other. They found that increasing the concentration of a cationic surfactant, which absorbs as a monolayer on the shearing surfaces, lowered the coefficient of friction (CoF). Valtiner et al. (2012) investigated the potential dependent friction forces between a mica surface and a gold electrode. They performed experiments measuring the friction on a gold surface maintained at $0 \mathrm{~V}$ vs. $\mathrm{Ag} / \mathrm{AgCl}$ and a positively charged gold surface $(+0.4 \mathrm{~V}$ vs. $\mathrm{Ag} / \mathrm{AgCl})$. The latter surface exhibited higher kinetic friction compared to the neutral gold surface. They attributed the increase in friction to the formation of a viscous water layer at the positively charged electrode. However, the increase in the friction was relatively small because of the limited range of applied positive potential. Based on these previous studies, it has been inferred that water molecules can reorient in the presence of an applied electric field which in turn can influence interfacial normal and friction forces.

In our previous work, we presented an exploratory study on the effect of an applied potential on the friction force between an AFM tip and a gold electrode surface over a wider range of applied potentials (i.e., -0.6 to $+0.6 \mathrm{~V}$ vs. Ag) (Pashazanusi et al., 2017b). We observed a reversible potential-dependent friction behavior on a gold electrode surface as the surface potential was increased. At $\sim+0.4 \mathrm{~V}$, a sharp increase in the friction forces was observed. The CoF between an AFM tip and a gold surface (see Figure 1) at the applied potential of $+0.6 \mathrm{~V}$ was reported to be 26 times higher than that at the applied potential of $-0.6 \mathrm{~V}$. The drastic change in the CoF was attributed to the formation of an interfacial water layer (IWL) on a positively charged electrode surface. However, there are several parameters in such electrochemical systems including the electrolyte concentration and composition that can possibly impact the properties and formation of the IWL.

This work is an extension of our previous work and provides a deeper understanding of how several experimental parameters (i.e., surface roughness, salt composition, and concentration) affect the tribological properties of a gold surface exposed to an applied electrical potential in an aqueous electrolyte solution. In addition, normal force measurements conducted at various approach velocities further support our hypothesis of the formation of a highly viscous water layer and are consistent with hydrodynamic drainage theory. These results provide a better understanding of the interactions between surfaces in the presence of an applied potential and can have a transformative impact in several areas of science including tribology, MEMs, energy storage devices, fuel cells, and catalysis.

\section{EXPERIMENTAL}

\section{Preparation of Gold Electrode}

Fifteen millimeter mica disks (Highest Grade V1 AFM Mica Disc) were purchased from Ted Pella, Inc. and utilized upon cleaving by a fresh sharp blade. Using thermal evaporation deposition (ATC Orion Sputtering System, AJA International, Inc.), the freshly cleaved mica was coated with a $5 \mathrm{~nm} \mathrm{Cr}$ film, acting as an adhesion layer, topped with a $100 \mathrm{~nm}$ gold film. A uniform smooth gold film was obtained by maintaining a slow deposition rate $(1 \AA / s)$ and a high vacuum $\left(\sim 10^{-6}\right)$. The substrate was allowed to cool down in the chamber before rinsing with distilled water and drying in air. AFM was used to characterize the surface roughness of the gold substrate $(\mathrm{rms} \sim 3.5 \mathrm{~nm})$ based on AFM images (Figure 2A).

\section{Preparation of Ultra-Smooth Gold Electrode}

Atomically smooth gold surfaces were prepared using the mica templating method (Chai and Klein, 2007). First, a $42 \mathrm{~nm}$ gold film on mica was deposited in an electron-beam evaporation system. A muscovite mica sheet of $10 \mathrm{~cm}$ in diameter and a few $100 \mu \mathrm{m}$ thick were freshly cleaved before the deposition to avoid any contamination. Second, pieces of $1.5 \times 1.5 \mathrm{~cm}^{2}$ step-free regions of the gold-coated mica sheet were cut and silicon wafer pieces were cut in squares of $1 \times 1 \mathrm{~cm}^{2}$. A few $100 \mu \mathrm{m}$ thick layer of UV-curing glue (Norland 81) was applied on top of each clean silicon wafers piece in a laminar flood hood environment before carefully place the gold-coated mica pieces with the gold side facing down on the glue. The UV glue was cured for at least $2 \mathrm{~h}$. Lastly, right before using the gold surface, the mica was peeled off the gold.

In both thermal evaporation and E-beam evaporation devices, a quartz crystal was used to calibrate, and control the deposition rate and the film thickness.

\section{In situ Electrochemistry-AFM}

A 3-electrode electrochemical cell was used to track changes in friction as a function of applied potential between the 


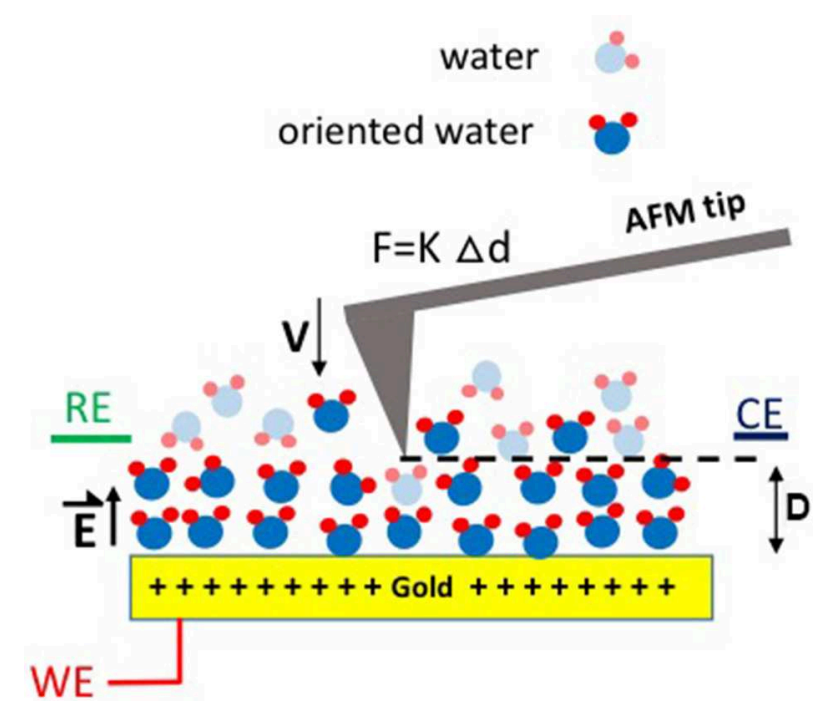

FIGURE 1 | Schematic illustration of the experimental setup used to measure the friction force $F$ between an AFM tip and a Au surface subject to applied potentials. RE, WE, and CE refer to the reference, working, and counter electrodes, respectively. $\mathrm{D}$ is the separation distance between the tip and the electrode surface, $\mathrm{V}$ is the approach velocity, $\mathrm{K}$ is the spring constant, and $\Delta \mathrm{d}$ is the deflection of the AFM tip cantilever, respectively. $\overrightarrow{\mathrm{E}}$ is the electric field generated as a result of the applied potential.

AFM tip and the gold surface. The potential range was limited so as to prevent the oxidation of gold. $\mathrm{A} \mathrm{Au}$ substrate, Pt mesh, and $\mathrm{Ag}$ wire served as the working, counter, and reference electrode, respectively. Electrochemical experiments were performed in different concentrations of aqueous $\mathrm{NaCl}$ (i.e., $1,10 \mathrm{mM}, 0.1$, and $1 \mathrm{M}$ ) to investigate the effect of salt concentration on friction forces. To explore the influence of ions on the potential-dependent friction, $0.1 \mathrm{M}$ aqueous $\mathrm{NaOH}$ was used as an alternative electrolyte. In order to confirm that the electrochemical setup in the AFM experiments was reliable, similar electrochemical experiments were performed using a conventional PTFE electrochemical cell (Oguntoye et al., 2017).

\section{Friction Measurements Using AFM}

Friction forces were measured using atomic force microscopy (AFM) (Dimension Icon, Bruker) in lateral force friction (LFM) mode. An AFM probe (NTESP, Bruker) with a sharp tip (estimated radius $10 \mathrm{~nm}$, height $15 \mu \mathrm{m}$, spring constant $\mathrm{K}_{\mathrm{c}}=$ $34 \mathrm{~N} / \mathrm{m}$ ) was used for the friction measurements on the gold substrates. Additionally, an AFM colloidal probe (CP-NCH-SiO$\mathrm{D}$, sQube, estimated diameter $10 \mu \mathrm{m}$, spring constant $\mathrm{K}_{\mathrm{c}}=37$ $\mathrm{N} / \mathrm{m}$ ) was used to measure force curves (i.e., normal forces as a function of separation distance between the colloidal probe and the gold surface).

The normal spring constant of the cantilever was calibrated by using the thermal tune technique in air and liquid. The lateral (or torsional) spring constant $\mathrm{k}_{\mathrm{T}}$ of the cantilever was obtained by using the following equation: $k_{T}=G w t^{3}\left(3 h^{2} l\right)$ where $G, w, t, l$ are the shear modulus, width, thickness, length of the cantilever, respectively, and $h$ is tip height (Cannara et al., 2006). The normal sensitivity of the cantilever was obtained via measuring the slope of the vertical voltage signal vs. distance curve (Palacio and Bhushan, 2010). The lateral sensitivity of the cantilever was calculated by averaging the initial slope of the friction loop, in which the lateral voltage signal is plotted vs. lateral distance, in both forward and backward scan directions (Liu et al., 1996; Cain et al., 2000).

Friction was measured by recording the lateral deflection of the cantilever as the tip scanned the surface perpendicular to long axis of the cantilever (scan size of $500 \mathrm{~nm}$ and speed of $1 \mu \mathrm{m} \mathrm{s}^{-1}$ ) while the electrical potential of the gold surface was varied. A method previously proposed in the literature (Schwarz et al., 1996) was used to convert the lateral voltage signal into quantitative friction forces. Friction loops were recorded from the same location.

\section{Normal Force Measurement Using AFM}

Atomic force microscopy Ramp mode was used to record normal forces vs. separation distance as a tip approaches the gold substrate followed by jumping into contact and then separates and retracts from the surface. To study the effect of the approach velocity on the normal force interactions, force-distance curves were obtained for approaching velocities of 50, 100, 200, 500, and $900 \mathrm{~nm} / \mathrm{s}$. A $200 \mathrm{~nm}$ ramp scan size was used in these experiments. The "Ramp mode" consists of the tip moving toward the surface at a pre-determined velocity until contact is achieved followed by separation at the same velocity. The total displacement, i.e., ramp size (which is correlated to the separation distance between the tip and the surface) can also be varied.

\section{RESULTS AND DISCUSSION}

Based on prior literature presented in the introduction and our past work (Pashazanusi et al., 2017b), we hypothesize that the underlying and dominant mechanism that modulates the friction force is hydrogen bonding between oriented IWLs and also between the IWLs and the AFM tip (and/or the hydration layer on the AFM tip). The results we present do not provide a direct evidence of the formation of IWLs, however they do support our hypothesis. Below we present our results and provide a discussion of the results with the assumption that hydrogen bonding is the underlying interaction that dominates this phenomenon. Our assumption is further supported by recent work which reproduced our results and further demonstrated that the ability of the tip to experience hydrogen bonding (i.e., a hydrophilic silicon oxide tip capable of hydrogen bonding showed a change in friction as a function of applied potential while a hydrophobic polystyrene tip not capable of hydrogen bonding did not experience any change in friction as a function of the applied potential) affected the friction forces ( $\mathrm{Li}$ et al., 2018). Figure 2A shows the friction force between a sharp AFM tip and a gold surface as a function of the applied normal load under four different conditions: (i) on dry gold, (ii) on gold under aqueous conditions with no applied potential [i.e., open circuit potential (OCP)], (iii) on gold under aqueous conditions 
A

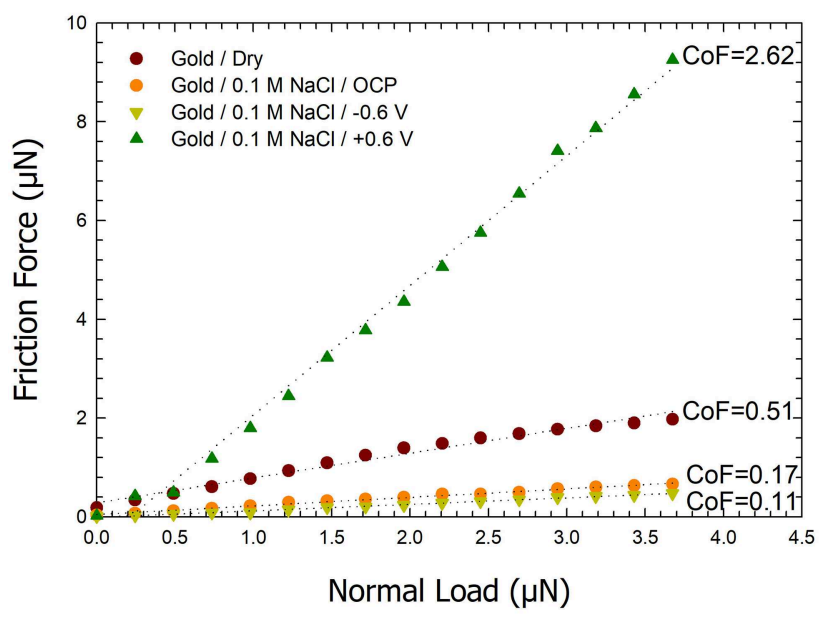

B

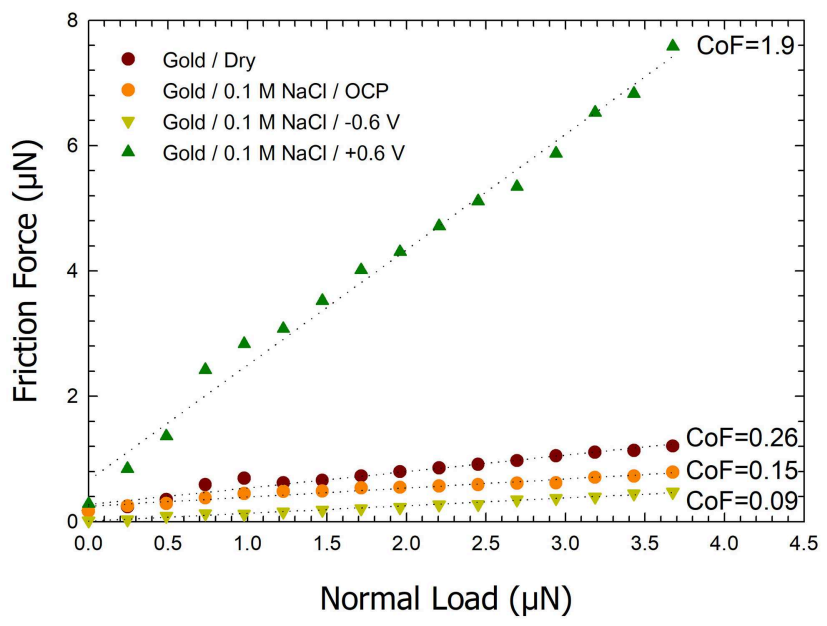

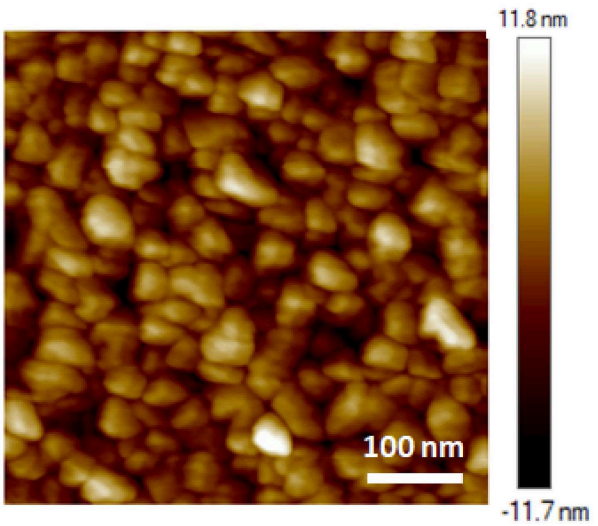

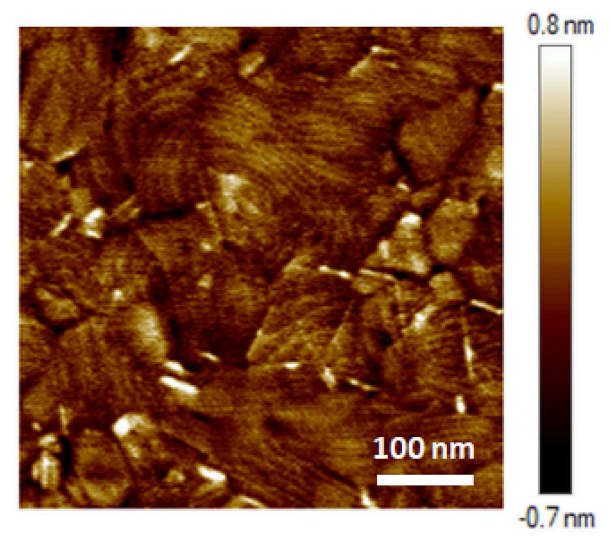

FIGURE 2 | Plots of the friction force vs. normal load for dry, open circuit, and applied potentials of -0.6 and $+0.6 \mathrm{~V}$ between a sharp AFM tip and (A) a gold surface formed by sputtering (B) an ultra-smooth Au surface formed by e-beam deposition. The images to the right are AFM height images of the corresponding gold surfaces. A scan size of $500 \mathrm{~nm}$ and scan speed of $1 \mu \mathrm{m} . \mathrm{s}^{-1}$ was used for friction measurements.

with a $-0.6 \mathrm{~V}$ vs. Ag potential, and (iv) on gold under aqueous conditions with a $+0.6 \mathrm{~V}$ vs. Ag potential. The friction force increases linearly with load in agreement with Amontons' law for all conditions. The CoF between the AFM tip and the gold surface under dry conditions is found to be $\sim 0.51$ which is in a similar range to previously reported values in the literatures (Antler, 1963). Under aqueous $\mathrm{NaCl}$ solution conditions and without applying a potential, the gold surface inherently gains a slight negative charge of $-0.12 \mathrm{~V}$ (Pashazanusi et al., 2017b) and the $\mathrm{CoF}$ is lowered to 0.17. Under these conditions, the aqueous salt solution serves as a boundary lubricant thereby reducing the interaction between the AFM tip and the Au surface. Hydrogen bonding between the AFM tip and the IWL is still present. By applying a negative potential on the Au surface, the number of broken hydrogen bonds increases (VelascoVelez et al., 2014; Pashazanusi et al., 2017b), which in turn decreases the attractive interaction between the tip and the IWLs. Thus, under aqueous conditions with a $-0.6 \mathrm{~V}$ applied potential, water behaves as a more effective boundary lubricant and lowers the friction further, resulting in a CoF of 0.11 . Upon applying a positive potential, e.g., $+0.6 \mathrm{~V}$, hydrogen bonding becomes more effective, resulting in water molecules next to the Au surface reorganizing into a nanostructured fluid and interacting strongly, again through hydrogen bonding, with the AFM tip. The formation of highly oriented water molecules is also expected to increase the IWL thickness, which will be discussed later. Under these conditions, the IWL no longer acts as an effective boundary lubricant, and results in a high CoF of 2.62.

The same experiment was conducted on an ultra-smooth gold surface to verify if the roughness of the gold surface affected the formation of the IWL. The ultra-smooth gold surface had an rms value of $0.2 \mathrm{~nm}$ (see Figure 2B) compared to the gold surfaces formed by sputtering with an rms of $3.3 \mathrm{~nm}$. Figure 2B shows a plot of friction force on the ultra-smooth gold surface vs. applied normal load under the same aforementioned 


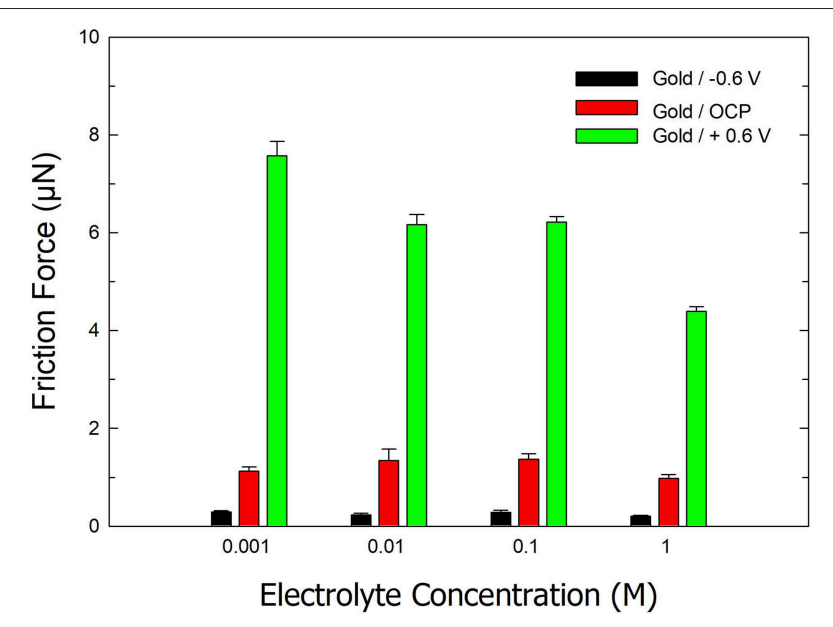

FIGURE 3 | Bar chart of the average friction force on ultra-smooth gold in electrolytes of various salt concentrations under open circuit and applied potentials of -0.6 and $+0.6 \mathrm{~V}$. Error bars indicate standard deviations from 10 repeats. The normal load is $2.5 \mu \mathrm{N}$.

conditions. Under dry conditions, the CoF on the ultra-smooth gold surface was 0.26 . Ultra-smooth gold contains smaller and fewer asperities thereby resulting in lower friction forces. Under aqueous conditions and an OCP, the CoF is measured as 0.15 . At the potential of $-0.6 \mathrm{~V}$, the coefficient the friction decreases to 0.09 . At the potential of the $+0.6 \mathrm{~V}$, the $\mathrm{CoF}$ is again observed to be high $(\mathrm{CoF}=1.9)$. Although the value of coefficient of the friction on the ultra-smooth gold surface is smaller than that observed on the gold sputtered surface, the trend of the change in friction as a function of the applied load and potential is similar. Both gold substrates exhibit high $\mathrm{CoF}$ values when a positive potential is applied but low $\mathrm{CoF}$ values when a negative potential is applied. Based on these results, and to avoid any influence of surface roughness, all future experiments described below were conducted on ultra-smooth Au surfaces. Based on our previous work (Pashazanusi et al., 2017b), we do not expect any damage to the gold surface under the normal loads used in this study.

Figure 3 displays the effect of electrolyte concentration on the friction forces on ultra-smooth gold under aqueous conditions and an (i) OCP, (ii) applied potential of $-0.6 \mathrm{~V}$, and (iii) applied potential of $+0.6 \mathrm{~V}$. The friction experiments were performed in electrolytes with concentrations of $1,10 \mathrm{mM}$, 0.1 , and $1 \mathrm{M}$. At a potential of $-0.6 \mathrm{~V}$, low friction values were obtained for all concentrations. Similarly, under aqueous conditions and no applied potential, the friction forces were similar for all concentrations. However, a decrease in friction was observed with an increase in electrolyte ionic strength at positive potentials. At an applied potential of $+0.6 \mathrm{~V}$, the friction force is $\sim 7.56 \pm 0.3 \mu \mathrm{N}$ in a $1 \mathrm{mM}$ electrolyte compared to $4.38 \pm 0.1$ $\mu \mathrm{N}$ in a $1 \mathrm{M}$ electrolyte. We attribute this decrease in friction to a similar mechanism that is involved in the freezing point depression of water in the presence of salt. In the latter case, salt ions disrupt the equilibrium of water molecules entering

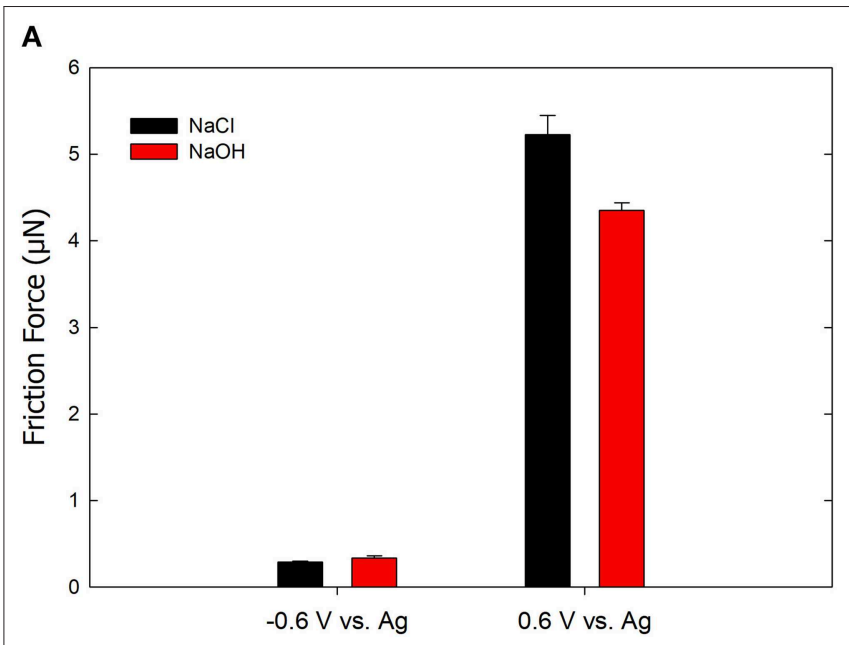

B
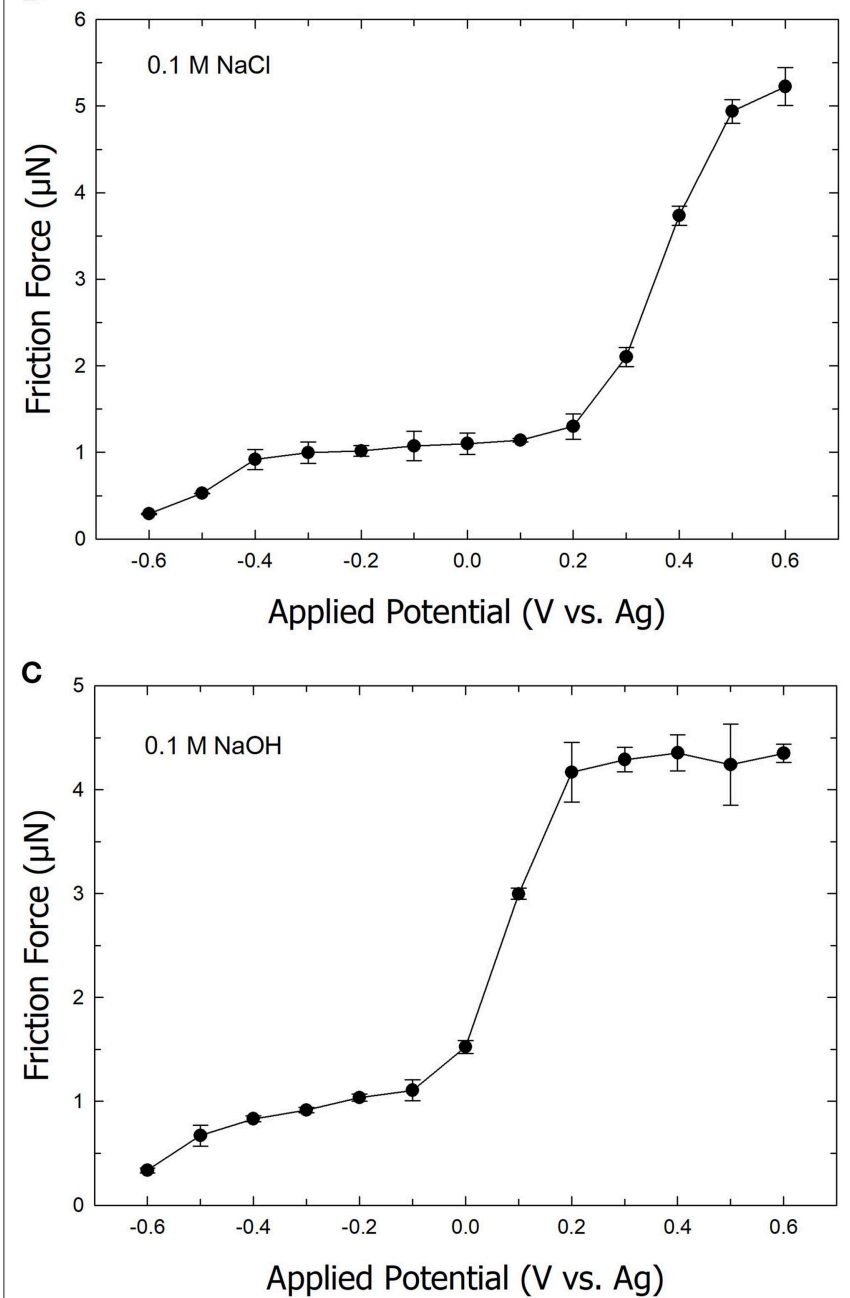

FIGURE 4 | (A) Bar chart of the friction force on ultra-smooth gold surface in an aqueous $0.1 \mathrm{M} \mathrm{NaCl}$ solution (black) and an aqueous $0.1 \mathrm{M} \mathrm{NaOH}$ solution (red). Plot of the friction force as a function of the applied potential in the range of -0.6 to $+0.6 \mathrm{~V}$ in (B) aqueous $\mathrm{NaCl}$ solution and (C) aqueous $\mathrm{NaOH}$ solution. Error bars indicate standard deviations from five repeats. The normal load is $2.5 \mu \mathrm{N}$. 
and leaving the solid state (i.e., ice). A similar mechanism is proposed to explain the decrease in friction forces with increasing salt concentration. The presence of salt disrupts the hydrogen bonding between the AFM tip surface and the IWLs thereby reducing friction.

To understand the effect of the electrolyte anion on the friction forces, friction experiments were performed using $0.1 \mathrm{M}$ $\mathrm{NaOH}$ as an electrolyte instead of $0.1 \mathrm{M} \mathrm{NaCl}$. Figure 4A shows the friction forces on positively and negatively charged $\mathrm{Au}$ surfaces using $\mathrm{NaOH}$ compared to $\mathrm{NaCl}$. At a potential of $-0.6 \mathrm{~V}$, the friction force is similar for both $\mathrm{NaOH}$ (red column) and $\mathrm{NaCl}$ (black column) electrolytes. At the potential of $+0.6 \mathrm{~V}$, the friction is observed to be higher in a $\mathrm{NaCl}$ electrolyte solution compared to a $\mathrm{NaOH}$ electrolyte solution. Figure 4B exhibits a plot of friction forces as a function of applied potential on an ultra-smooth gold surface using $\mathrm{NaCl}$ as the electrolyte. At the applied potential of $-0.6 \mathrm{~V}$, the friction is at its lowest value. As the applied potential increases to $+0.2 \mathrm{~V}$, the friction gradually increases. Further increase in the applied potential (i.e., $>+0.2 \mathrm{~V}$ ) leads to a sharp increase in the friction force. In our previous work, we demonstrated that the potential range of -0.6 to $+0.6 \mathrm{~V}$, where main changes in friction forces occur, is free of any Faradaic process including gold oxidation (Pashazanusi et al., 2017b). The friction of the ultra-smooth gold drastically increases from a value of $0.2 \mu \mathrm{N}$ (at $-0.6 \mathrm{~V}$ ) to $5.2 \mu \mathrm{N}$ (at $+0.6 \mathrm{~V})$ corresponding to an increase in friction by a factor of 26. The sharp changes in the friction can be explained by the dependency of the shear viscosity of interfacial water (which is dependent on the number of hydrogen bonds) at the gold interface. These results suggest that the reorientation of water molecules at the interface forms a highly viscous IWL at the positively charged gold surface, which increases the shear viscosity, thereby increasing friction. However, at the negatively charged surface, the shear viscosity of water next to the gold surface becomes smaller due to lower number of hydrogen bonds, resulting in a decrease in friction.

Figure 4C shows data for the same experiment as above with the exception of substituting $\mathrm{NaCl}$ with $\mathrm{NaOH}$ as the electrolyte. The friction is again observed to be at the lowest at a potential of $-0.6 \mathrm{~V}$, followed by a gradual increase in friction as the potential increases. Similarly, a sharp rise in friction is found over the range of 0 to $+0.2 \mathrm{~V}$. The friction then levels off as the applied potential is increased to $+0.6 \mathrm{~V}$. The critical potential, where the drastic change in friction is observed, shifts to a lower value when $\mathrm{NaOH}$ is used as the electrolyte. The observed shift in the critical potential can be attributed to differences between the inhibition of the hydrogen bond formation by $\mathrm{Cl}^{-}$ions compared to $\mathrm{OH}^{-}$ ions. Because of the self-ionization of water (i.e., $\mathrm{H}_{2} \mathrm{O}+\mathrm{H}_{2} \mathrm{O} \leftrightarrow$ $\mathrm{H}_{3} \mathrm{O}^{+}+\mathrm{OH}^{-}$), the addition of $\mathrm{OH}^{-}$(i.e., the increase in $\mathrm{pH}$ as a result of using $\mathrm{NaOH}$ ) is not expected to significantly disrupt hydrogen bonding or the formation of a viscous IWL. However, $\mathrm{Cl}^{-}$ions disrupt hydrogen bond formation, which manifests as requiring a higher positive potential (or driving force) to form the viscous IWL. The difference in the equilibrium potential between a $\mathrm{Ag}$ wire in $0.1 \mathrm{M} \mathrm{NaOH}$ compared to $0.1 \mathrm{M} \mathrm{NaCl}$ was measured and found to be negligible. Therefore, the difference in tribological behavior between the two different electrolyte systems cannot be attributed to the reference electrode. Another feature that is apparent when comparing Figures $\mathbf{4 B}, \mathbf{C}$ is the fact the friction force levels at a value of $4.3 \mu \mathrm{N}$ in the case of using $\mathrm{NaOH}$ compared to a still increasing value beyond $5 \mu \mathrm{N}$ in the case of using $\mathrm{NaCl}$, at high positive potentials. The latter would
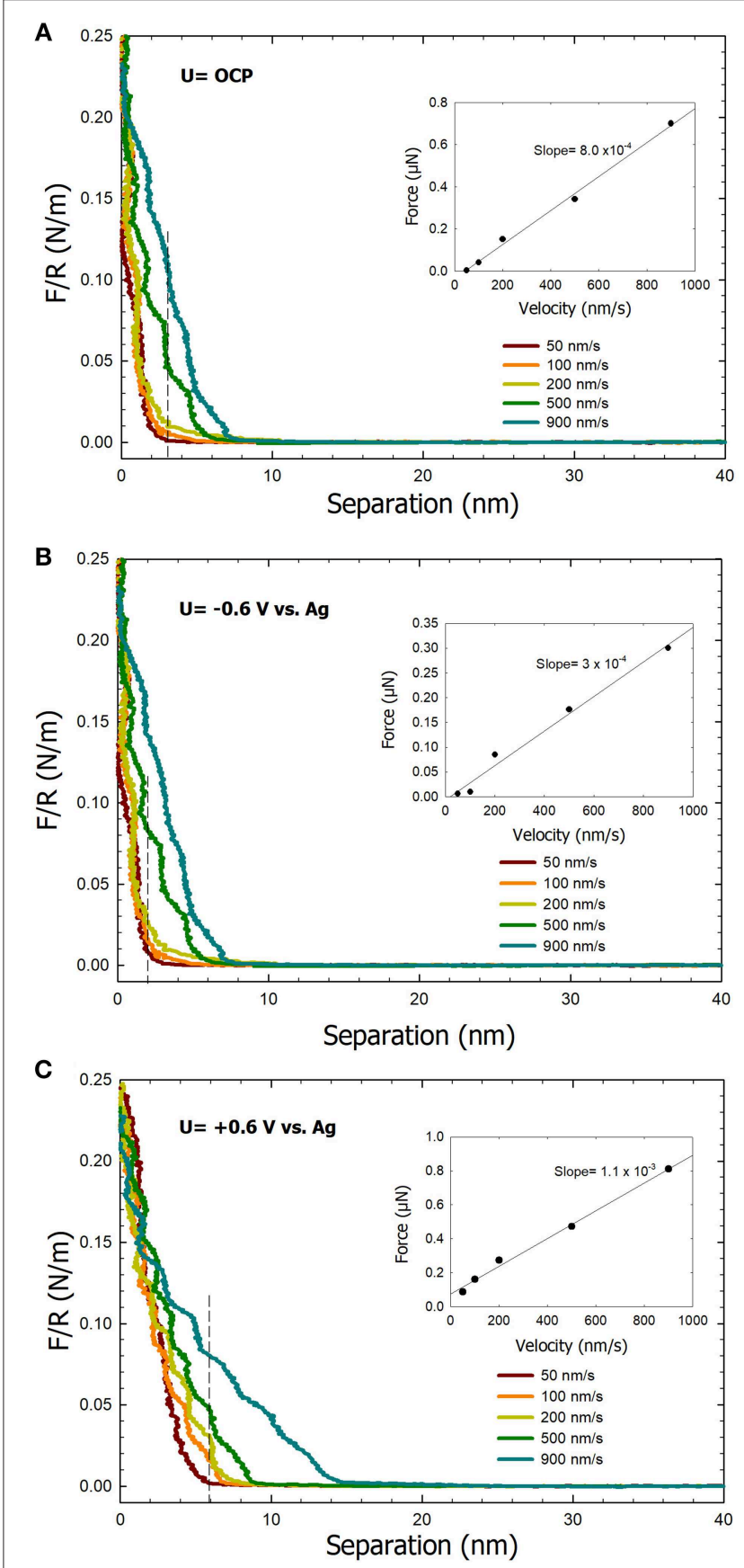

FIGURE 5 | Normal force curves recorded between a colloidal AFM probe and an ultra-smooth gold electrode surface at the various tip approach velocities in $0.1 \mathrm{M} \mathrm{NaCl}$ as the electrolyte at (A) open circuit potential, (B) $-0.6 \mathrm{~V}$, and (C) $+0.6 \mathrm{~V}$. The inset plots correspond to force vs. velocity data at a specific separation distance (denoted by the dashed lines). 
imply that the AFM tip experiences stronger interactions with the IWL in the presence of $\mathrm{NaCl}$ compared to $\mathrm{NaOH}$. We attribute this difference to the fact that at a high $\mathrm{pH}$ (i.e., in the presence of $0.1 \mathrm{M} \mathrm{NaOH}$ ), the hydroxyl groups present on the AFM tip are de-pronated, thereby yielding a larger negative charge on the AFM tip (Tourinho et al., 2002). As a result, hydrogen bonding is disrupted (similar to when a negative potential is applied to a gold surface) at the IWL/AFM tip interface, which results in a decrease in the magnitude of the friction force.

Figure 5 shows the effect of the approach velocity on the normal force between a colloidal AFM tip probe and an ultrasmooth gold substrate. Experiments were performed under open circuit, an applied potential of $-0.6 \mathrm{~V}$, and an applied potential of $+0.6 \mathrm{~V}$, using approach velocities of $50,100,200,500$, and $900 \mathrm{~nm} / \mathrm{s}$ in $0.1 \mathrm{M} \mathrm{NaCl}$. A repulsive force regime is observed in the force curves under both open circuit and the applied potential of $+0.6 \mathrm{~V}$ conditions (Figures 5A,C, respectively) that can be attributed to the presence of a highly viscous IWL. Under these conditions, a noticeable difference in the forces curves is observed specifically with the onset of the repulsive force. Under the applied potential of $-0.6 \mathrm{~V}$, the repulsive force is negligible at the small velocities, which is consistent with our previous work (Pashazanusi et al., 2017b). However, upon increasing the velocity to $900 \mathrm{~nm} / \mathrm{s}$, a small repulsive force regime appears in the force curves (Figure 5B). The classical hydrodynamic drainage model $F=6 \pi \eta \mathrm{R}^{2} \mathrm{~V} / \mathrm{D}$, where $\eta$ is the effective viscosity, $\mathrm{R}$ is the tip radius, $\mathrm{V}$ is the approach velocity, and $\mathrm{D}$ is the separation distance between the tip and surface (Feibelman, 2004) predicts a linear dependence of the force $F$ with increasing velocity. The insets in Figure 5 show plots of the normal force as a function of the approach velocity. The forces are reported at separation distances of 2, 4, and 5.5 (dotted lines) and obtained from the force curves in Figure 5. These specific separation distances were chosen for our analysis based on when a repulsive force became significant in the force curves. In all three cases, a linear dependence between the normal force and the approach velocity was obtained which further supports the formation of a highly viscous IWL. Electrical double layer forces are negligible in our system due to the high ionic strength of the electrolyte (i.e., $0.1 \mathrm{M} \mathrm{NaCl}$ resulting in a Debye length of $\sim 1 \mathrm{~nm}$ ) (Israelachvili, 2011). A linear regression of the normal force vs. approach velocity data yields a slope that correlates with the viscosity at a constant separation distance. The linear plotted graph under applied potential of $+0.6 \mathrm{~V}$ condition exhibits the highest slope, thereby the highest viscosity. We note that the approach velocity in the hydrodynamic drainage model is the instantaneous velocity which cannot be directly measured with AFM (due to the deflection of the cantilever), however, we assume that the instantaneous velocity is close to

\section{REFERENCES}

Achanta, S., and Celis, J. P. (2015). "Nanotribology of mems/nems," in Fundamentals of Friction and Wear on the Nanoscale (New York, NY: Springer), 631-656. doi: 10.1007/978-3-319-10560-4_27 the applied approach velocities. The observed step-like behavior in the force curves is reminiscent of the oscillatory forces first observed by Israelachvili and Pashley (1983) because of the molecular ordering of water at surfaces resulting in repulsive hydration forces. Previously, it has been shown that the viscosity of interfacial water next to the gold with the surface potential of $+0.6 \mathrm{~V}$ is $\sim 5$ orders of magnitude higher than for bulk water (Guriyanova et al., 2011). At a surface potential of $-0.6 \mathrm{~V}$, the viscosity of the IWL next to the gold surface deceases to lower values (Park, 2011; Pashazanusi et al., 2017b), even lower compared to the OCP case. The latter is again consistent with the fact that in the OCP case, water still orients to a certain extent with relatively more effective hydrogen bonding compared to bulk water.

\section{CONCLUSIONS}

In this study, the effect of several system parameters (i.e., surface roughness, salt concentration, and composition) on the friction force between an AFM tip and a gold surface was investigated. Applying a positive potential to a gold surface, irrespective of surface roughness, resulted in high friction forces between an AFM tip and the gold surface. We attribute the increase in friction to the effective hydrogen bonding between the viscous IWL and the AFM tip. However, interfacial water next to a gold surface with an anodic applied potential has properties closer to bulk water, which resulted in lower friction. Higher friction forces were observed when using electrolyte solutions of lower ionic strength when a potential of $+0.6 \mathrm{~V}$ was applied on the $\mathrm{Au}$ surface. Additionally, it was found the sharp rise in friction was still present when using $\mathrm{NaOH}$ instead of $\mathrm{NaCl}$ as the aqueous electrolyte albeit the sharp rise occurred at a different critical applied potential. Force curve measurements performed at different approach velocities yielded a linear dependence, which is consistent with the hydrodynamic drainage model, and supported the formation of a highly viscous IWL at positive applied potential.

\section{DATA AVAILABILITY}

All datasets generated for this study are included in the manuscript and/or the supplementary files.

\section{AUTHOR CONTRIBUTIONS}

LP and SL designed and performed the experiments. KK provided samples and offered valuable suggestions for the experimental section. NP and YT designed the experiments and provided guidance for the project. 
Balzer, B. N., Gallei, M., Hauf, M. V., Stallhofer, M., Wiegleb, L., Holleitner, A., et al. (2013). Nanoscale friction mechanisms at solid-liquid interfaces. Angewandte Chemie. 52, 6541-6544. doi: 10.1002/anie.201301255

Binggeli, M., Christoph, R., Hintermann, H.-E., Colchero, J., and Marti, O. (1993). Friction force measurements on potential controlled graphite in an electrolytic environment. Nanotechnology 4, 59-63. doi: 10.1088/0957-4484/ $4 / 2 / 001$

Cain, R. G., Biggs, S., and Page, N. W. (2000). Force calibration in lateral force microscopy. J. Colloid Interface Sci. 227, 55-65. doi: 10.1006/jcis.2000.6840

Cannara, R. J., Eglin, M., and Carpick, R. W. (2006). Lateral force calibration in atomic force microscopy: a new lateral force calibration method and general guidelines for optimization. Rev. Sci. Instr. 77:053701. doi: 10.1063/1. 2198768

Carpick, R. W., and Salmeron, M. (1997). Scratching the surface: fundamental investigations of tribology with atomic force microscopy. Chem. Rev. 97, 1163-1194. doi: 10.1021/cr960068q

Chai, L., and Klein, J. (2007). Large area, molecularly smooth (0.2 nm rms) gold films for surface forces and other studies. Langmuir 23, 7777-7783. doi: $10.1021 / \mathrm{la} 0637380$

Dhinojwala, A., and Granick, S. (1997). Relaxation time of confined aqueous films under shear. J. Am. Chem. Soc. 119, 241-242. doi: 10.1021/ja9632318

Dhopatkar, N., Defante, A. P., and Dhinojwala, A. (2016). Ice-like water supports hydration forces and eases sliding friction. Sci. Adv. 2:e1600763. doi: $10.1126 /$ sciadv. 1600763

Feibelman, P. J. (2004). Effect of high-viscosity interphases on drainage between hydrophilic surfaces. Langmuir 20, 1239-1244. doi: 10.1021/la035430s

Gallagher, P., Lee, M., Amet, F., Maksymovych, P., Wang, J., Wang, S., et al. (2016). Switchable friction enabled by nanoscale self-assembly on graphene. Nat. Commun. 7:10745. doi: 10.1038/ncomms10745

Greiner, C., Felts, J. R., Dai, Z., King, W. P., and Carpick, R. W. (2012). Controlling nanoscale friction through the competition between capillary adsorption and thermally activated sliding. ACS Nano. 6, 4305-4313. doi: 10.1021/nn $300869 \mathrm{w}$

Guriyanova, S., Mairanovsky, V. G., and Bonaccurso, E. (2011). Superviscosity and electroviscous effects at an electrode/aqueous electrolyte interface: An atomic force microscope study. J. Colloid Interface Sci. 360, 800-804. doi: $10.1016 /$ j.jcis.2011.04.072

Hugel, T., and Seitz, M. (2001). The study of molecular interactions by AFM force spectroscopy. Macromol. Rapid Commun. 22, 989-1016. doi: 10.1002/15213927(20010901)22:13<989::AID-MARC989>3.0.CO;2-D

Israelachvili, J. N. (2011). Intermolecular and Surface Forces. Amsterdam: Academic press.

Israelachvili, J. N., and Pashley, R. M. (1983). Molecular layering of water at surfaces and origin of repulsive hydration forces. Nature 306, 249-250. doi: $10.1038 / 306249 \mathrm{a} 0$

Karuppiah, K. S., Zhou, Y., Woo, L. K., and Sundararajan, S. (2009). Nanoscale friction switches: friction modulation of monomolecular assemblies using external electric fields. Langmuir 25, 12114-12119. doi: 10.1021/la $901221 \mathrm{~g}$

Khan, S. H., Matei, G., Patil, S., and Hoffmann, P. M. (2010). Dynamic solidification in nanoconfined water films. Phys. Rev. Lett. 105:106101. doi: 10.1103/PhysRevLett.105.106101

Kim, H.-J., and Kim, D.-E. (2009). Nano-scale friction: a review. Int. J. Prec. Eng. Manufact. 10, 141-151. doi: 10.1007/s12541-009-0039-7

Kim, H. I., Kushmerick, J. G., Houston, J. E., and Bunker, B. C. (2003). Viscous "interphase" water adjacent to oligo (ethylene glycol)-terminated monolayers. Langmuir 19, 9271-9275. doi: 10.1021/la034585x

Kim, S. H., Asay, D. B., and Dugger, M. T. (2007). Nanotribology and MEMS. Nano Today 2, 22-29. doi: 10.1016/S1748-0132(07)70140-8

Labuda, A., Hausen, F., Gosvami, N. N., Grütter, P. H., Lennox, R. B., and, Bennewitz, R. (2011). Switching atomic friction by electrochemical oxidation. Langmuir 27, 2561-2566. doi: 10.1021/la104497t

Lee, S. H., and Rossky, P. J. (1994). A comparison of the structure and dynamics of liquid water at hydrophobic and hydrophilic surfaces-a molecular dynamics simulation study. J. Chem. Phys. 100, 3334-3345. doi: 10.1063/1. 466425

Li, S. W., Bai, P. P., Li, Y. Z., Chen, C. F., Meng, Y. G., and Tian, Y. (2018). Electric potential-controlled interfacial interaction between gold and hydrophilic/hydrophobic surfaces in aqueous solutions. J. Phys. Chem. C. 122, 22549-22555. doi: 10.1021/acs.jpcc.8b06755

Liu, E., Blanpain, B., and Celis, J.-P. (1996). Calibration procedures for frictional measurements with a lateral force microscope. Wear 192, 141-150. doi: 10.1016/0043-1648(95)06784-1

Meyer, E., Heinzelmann, H., Grütter, P., Jung, T., Hidber, H.-R., Rudin, H., and Güntherodt, H.-J. (1989). Atomic force microscopy for the study of tribology and adhesion. Thin Solid Films 181, 527-544. doi: 10.1016/0040-6090(89)90522-1

Mo, Y., Turner, K. T., and Szlufarska, I. (2009). Friction laws at the nanoscale. Nature 457, 1116-1119. doi: 10.1038/nature07748

Oguntoye, M., Oak, S., Pashazanusi, L., Pratt, L., and Pesika, N. S. (2017). Vertically-aligned carbon nanotube arrays as binder-free supports for nickel cobaltite based faradaic supercapacitor electrodes. Electrochim. Acta 236, 408-416. doi: 10.1016/j.electacta.2017.03.172

Overney, R., and Meyer, E. (1993). Tribological investigations using friction force microscopy. MRS Bull. 18, 26-34. doi: 10.1557/S088376940 0047096

Palacio, M. L., and Bhushan, B. (2010). Normal and lateral force calibration techniques for AFM cantilevers. Crit. Rev. Solid State Mater. Sci. 35, 73-104. doi: 10.1080/10408430903546691

Park, J. Y. (2011). Tuning nanoscale friction on Pt nanoparticles with engineering of organic capping layer. Langmuir 27, 2509-2513. doi: 10.1021/la $104353 \mathrm{f}$

Park, J. Y., Ogletree, D. E., Thiel, P. A., and Salmeron, M. (2006). Electronic control of friction in silicon pn junctions. Science 313, 186-186. doi: 10.1126/science.1125017

Pashazanusi, L., Lwoya, B., Oak, S., Khosla, T., Albert, J. N. L., Tian, Y., et al. (2017a). Enhanced Adhesion of mosquitoes to rough surfaces. ACS Appl. Mater. Interfaces 9, 24373-24380. doi: 10.1021/acsami.7b06659

Pashazanusi, L., Oguntoye, M., Oak, S., Albert, J. N. L., Pratt, L. R., and Pesika, N. S. (2017b). Anomalous potential-dependent friction on $\mathrm{Au}$ (111) measured by AFM. Langmuir 34, 801-806. doi: 10.1021/acs.langmuir.7b03023

Pérez, R. (2014). Nanoscale friction: distorted by the tip. Nat. Mater. 13, 118-119. doi: $10.1038 /$ nmat3875

Plausinaitis, D., Pulmanas, A., Kubilius, V., Raudonis, R., and Daujotis, V. (2014). Properties of an interfacial solution layer at gold electrode surface in perchlorate and chloride solutions: piezoelectric resonator and drag force study. Electrochim. Acta 121, 278-284. doi: 10.1016/j.electacta.2014. 01.007

Raviv, U., and Klein, J. (2002). Fluidity of bound hydration layers. Science 297, 1540-1543. doi: 10.1126/science.1074481

Schirmeisen, A., and Schwarz, U. D. (2009). Measuring the friction of nanoparticles: a new route towards a better understanding of nanoscale friction. ChemPhysChem 10, 2373-2382. doi: 10.1002/cphc. 200900378

Schwarz, U., Köster, P., and Wiesendanger, R. (1996). Quantitative analysis of lateral force microscopy experiments. Rev. Sci. Instr. 67, 2560-2567. doi: $10.1063 / 1.1147214$

Strelcov, E., Kumar, R., Bocharova, V., Sumpter, B. G., Tselev, A., and Kalinin, S. V. (2015). Nanoscale lubrication of ionic surfaces controlled via a strong electric field. Sci. Rep. 5:8049. doi: 10.1038/srep08049

Sweeney, J., Hausen, F., Hayes, R., Webber, G. B., Endres, F., Rutland, M. W., et al. (2012). Control of nanoscale friction on gold in an ionic liquid by a potential-dependent ionic lubricant layer. Phys. Rev. Lett. 109:155502. doi: 10.1103/PhysRevLett.109.155502

Toney, M. F., Howard, J. N., Richer, J., Borges, G. L., Gordon, J. G., Melroy, O. R., et al. (1994). Voltage-dependent ordering of water molecules at an electrode-electrolyte interface. Nature 368:444. doi: 10.1038/ $368444 \mathrm{a} 0$

Tourinho, F. A., Campos, A. F. C., Aquino, R., Lara, M. C. F. L., da Silva, G., and Depeyrot, J. (2002). Surface charge density determination in electric double layered magnetic fluids. Braz. J. Phy. 32, 501-508. doi: 10.1590/S0103-97332002000300008

Valtiner, M., Banquy, X., Kristiansen, K., Greene, G. W., and Israelachvili, J. N. (2012). The electrochemical surface forces apparatus: the effect of surface roughness, electrostatic surface potentials, and anodic oxide growth on interaction forces, and friction between dissimilar surfaces 
in aqueous solutions. Langmuir 28, 13080-13093. doi: 10.1021/la 3018216

Velasco-Velez, J.-J., Wu, C. H., Pascal, T. A., Wan, L. F., Guo, J., Prendergast, D., (2014). The structure of interfacial water on gold electrodes studied by X-ray absorption spectroscopy. Science 346, 831-834. doi: 10.1126/science. 1259437

Weymouth, A. J., Hofmann, T., and Giessibl, F. J. (2014). Quantifying molecular stiffness and interaction with lateral force microscopy. Science 343, 1120-1122. doi: 10.1126/science.1249502

Xie, G., Luo, J., Liu, S., Guo, D., and Zhang, C. (2009). "Freezing” of nanoconfined fluids under an electric field. Langmuir 26, 1445-1448. doi: 10.1021/la903419v
Conflict of Interest Statement: The authors declare that the research was conducted in the absence of any commercial or financial relationships that could be construed as a potential conflict of interest.

Copyright (c) 2019 Pashazanusi, Kristiansen, Li, Tian and Pesika. This is an openaccess article distributed under the terms of the Creative Commons Attribution License (CC BY). The use, distribution or reproduction in other forums is permitted, provided the original author(s) and the copyright owner(s) are credited and that the original publication in this journal is cited, in accordance with accepted academic practice. No use, distribution or reproduction is permitted which does not comply with these terms. 